\title{
Circular RNA circ-PAN3 (hsa_circ_0100181) Promotes Hepatocellular Carcinoma Growth Through Sponging miR-153 to Elevate Cyclin D1 Expression
}

\section{Shuo Yu}

Tongji Hospital of Tongji Medical College of Huazhong University of Science and Technology

\section{Min Wang}

Tongji Hospital of Tongji Medical College of Huazhong University of Science and Technology

\section{Xu Li}

Tongji Hospital of Tongji Medical College of Huazhong University of Science and Technology

\section{Xingjun Guo}

Tongji Hospital of Tongji Medical College of Huazhong University of Science and Technology

Renyi Qin ( $\square$ qyinrendoc@163.com )

Department of Biliary-Pancreatic Surgery, Affiliated Tongji Hospital, Tongji Medical College, Huazhong University of Science and Technology, 1095 Jiefang Ave, 430030, Wuhan, Hubei Province, China https://orcid.org/0000-0002-5101-5259

\section{Primary research}

Keywords: Hepatocellular carcinoma, circ-PAN3, miR-153, RNA sponge

Posted Date: August 21st, 2020

DOI: https://doi.org/10.21203/rs.3.rs-62226/v1

License: (9) This work is licensed under a Creative Commons Attribution 4.0 International License. Read Full License 


\section{Abstract}

Background: Circular RNAs (circRNAs) are engaged in hepatocellular carcinoma (HCC) progression, but the mechanisms remain to be elucidated. This study aimed to unveil the expression pattern and potential biological mechanisms of a newly indentified circRNA, circ-PAN3, in HCC.

Methods: Cell Counting Kit-8 (CCK-8) assay and colony formation assay were used to assess cell proliferation. Transcription-quantitative PCR (RT-qPCR) analysis and western blot analysis were used to determine the relative expression level of mRNA and protein, respectively. Cell apoptosis assay was used to evaluate the apoptosis rate of transfected cells. Circlnteractome and Targetscan were utilized to predict the possible targets of circRNAs and miRNAs, respectively. Luciferase reporter assay and RNA pull-down assay were used to assess the direct interaction of RNAs. HCC cancer xenograft model was used to evaluate the biological process of circ-PAN3 in vivo. Student's $t$ test, $\chi^{2}$ test or one-way ANOVA was adopted appropriately.

Results: Circ-PAN3 was elevated in HCC tissues, and patients with high Circ-PAN3 expression had a poor survival outcome. Knockdown of circ-PAN3 expression suppressed cell viability, colony formation and cell proliferation in vitro and in vivo. Circ-PAN3 elevates cyclin D1 expression to promote HCC progression. Subsequently, using Circlnteractome, miR-153 were confirmed to interact with circ-PAN3 and was downregulated by circ-PAN3. Further, using Targetscan, cyclin D1 was validated to interact with miR-153 and was downregulated by miR-153. Addition of miR-153 expression with corresponsive mimics significantly reduced the expression of cyclin D1. Notably, the inhibition of cell viability, colony formation and proliferation induced by knockdown of circ-PAN3 were recovered following the combination with miR153 inhibitor, cyclin D1, respectively.

Conclusion: Together, this study demonstrated that a novel circ-PAN3/miR-153/cyclin D1 axis regulatory axis that promoted HCC progression.

\section{Introduction}

Hepatocellular carcinoma (HCC) is one of the most common malignancies in the world [1, 2]. Due to limited diagnostic methodologies, most patients are diagnosed at an advanced stage and are ineligible for liver transplantation or surgical resection $[3,4]$. Besides, this malignancy often exhibited high aggressiveness, invasiveness and frequent recurrence after resection [3]. As a result, the prognosis of HCC remains unsatisfactory [1]. It is urgent to investigate the detailed mechanism contributing to the initiation and progression of $\mathrm{HCC}$ in order to discover novel therapeutic targets for HCC patients.

Accumulating evidences showed that circular RNAs (circRNAs) are normally produced by scrambling of exons at the splicing process and are recognized as a novel class of endogenous noncoding RNA [5, 6]. CircRNAs are highly conserved and are characterized as covalently closed loop structures with neither a $5^{\prime}$ to 3' polarity nor a polyadenylated tail; they are RNA transcripts generated by the back-splicing of a single pre-mRNA and have gene-regulatory potential. Recently, studies have revealed that several circRNAs were 
abnormally expressed in HCC tissues and correlated with disease progression and prognosis [7-9]. However, the actual role of circRNA in HCC remains controversial. While several studies demonstrated an inhibitory role of circRNA in HCC [8], various studies also indicated a promotive role of circRNA [7]. Previous studies demonstrated that circ_0091570 could sponge miR-1307 to suppress the progression of HCC [8]. CircMTO1 could suppress HCC progression through miR-9/p-21 axis [10]. Recent studies elucidated that circSLC3A2 promoted HCC by sponging miR-490-3p and regulating PPM1F expression [11]. Recently, a newly circ-PAN3 was shown to be involved in drug resistance in acute myeloid leukemia and self-renewal of intestinal stem cells $[12,13]$. However, its role in HCC remains unknown. Therefore, the specific roles and potential mechanisms of circRNA in HCC remains elucidated further.

Cyclin D1 is a key cell cycle regulator, which plays a key role in completion of the G1/S transition in mammalian cells [14]. Multiple studies have demonstrated that aberrant expression of cyclin D1 induced various oncogenic responses and was associated with shorter survival outcome [15]. Previous studies reported that cyclin D1 could be positively regulated by X-linked inhibitor of apoptosis protein (XIAP) through E3 ligase-mediated phosphorylation [16]. Interestingly, circ-PAN3 could regulate positively XIAP to induce chemo-resistance in acute myeloid leukemia [17]. Although both circ-PAN3 and cyclin D1 are upregulated in several malignancies, the possible role of circ-PAN3 in the regulation of cyclin D1 remains largely unknow.

In the present study, we aimed to explore the role of circ-PAN3 in HCC. The data from the present study revealed that circ-PAN3 promoted HCC cell proliferation by regulating cyclin D1 through modulating miR153. The current study is the first to suggest that circ-PAN3 could promote HCC progression through circPAN3/miR-153/ cyclin D1 regulatory axis. Circ-PAN3 may be utilized as a promising diagnostic and therapeutic target in HCC patients.

\section{Methods}

\section{Ethics, patients and human tissues}

The protocol of this study was reviewed and approved by the Ethics Committee of Medical Research, Affiliated Tongji Hospital, Tongji Medical College, Huazhong University of Science and Technology. Patients provided written informed consent for the use of tumor and adjacent tissues at Affiliated Tongji Hospital, Tongji Medical College, Huazhong University of Science and Technology.

\section{Cell lines and reagents}

All the HCC cell lines including Huh7, HCCLM3 and SK-HEP-1 and the human normal liver cell line LO2 were purchased from Cell Bank of the Chinese Academy of Sciences (Chinese Academy of Sciences, Shanghai, China). Cells were cultured in DMEM (Invitrogen; Thermo Fisher Scientific, Inc.) containing 10\% fetal bovine serum (Invitrogen; Thermo Fisher Scientific, Inc.), penicillin (100 U/ml; Invitrogen; Thermo Fisher Scientific, Inc.) and streptomycin ( $100 \mathrm{U} / \mathrm{ml}$; Invitrogen; Thermo Fisher Scientific, Inc.). All cells were cultivated in a humidified incubator (Thermo Fisher Scientific, Inc.) at $37^{\circ} \mathrm{C}$ with $5 \% \mathrm{CO}_{2}$. 


\section{Cell transfection.}

HCC cells were planted in six-well plates and cultivated to $60-70 \%$ confluency for transfection using Lipofectamine $^{\mathrm{TM}} 3000$ (cat. no. L3000008; Invitrogen; Thermo Fisher Scientific, Inc.), according to the manufacturer's instructions. The sequences of the transfected mimics, inhibitors and small interfering RNA (siRNA) were as follows: si-circ-PAN3-1: 5'-UCUGACCCAAAACAACCCCdTdT-3'; si-circ-PAN3-2: 5'GAGAAAGUGGGGAAUGUCGUUdTdT-3'; si-NC: 5'- UUCUCCGAACGUGUCACGU -3'; miR-153 mimics: 5'UUGCAUAGUCACAAAAGUGAUC-3', miR-153 inhibitor: 5'-GAUCACUUUUGUGACUAUGCAA-3'.

\section{RNA extraction and reverse transcription-quantitative PCR (RT-qPCR) analysis.}

RNA from HCC cell lines or normal liver cell was isolated using TRIzol® reagent (Invitrogen; Thermo Fisher Scientific, Inc.). Then, PrimeScript RT master mix (cat. no. RR036A; Takara Bio, Inc.) was used to reverse-transcribe RNA (1000 ng) into CDNA according to manufacturer's protocol. qPCR was conducted using the SYBR Premix Ex Taq II kit (cat. no. DRR081A; Takara Bio, Inc.) with the LightCycler 96 system (Roche Diagnostics $\mathrm{GmbH}$ ). Primer pairs used for the qPCR were as follows: circ-PAN3: Forward: CGGAATTCGAATG, Reverse: CGGGATCCCCCACTTTCTC; miR-153: Forward: TTGCATAGTCACAAAAGTGAT, Reverse: CAGTGCGTGTCGTGGAGT; Cyclin D1: Forward: CCTGTCCTACTACCGCCTCA, Reverse: TCCTCCTCTTCCTCCTCCTC; GAPDH: Forward: CGGAGTCAACGGATTTGGTCGTAT, Reverse: AGCCTTCTCCATGGTGGTGAAGAC. GAPDH was utilized as the internal control for normalization.

\section{Cell proliferation assay.}

Cell proliferation was assessed with Cell Counting Kit-8 (CCK-8) assay (Dojindo Molecular Technologies, Inc.). After transfection, cells were seeded in 96 -well plates with a density of $2 \times 10^{3}$ cells/well. After cultured for 24,48 and $72 \mathrm{~h}$ at $37^{\circ} \mathrm{C}$, CCK-8 solution (10 $\mu$ l) was supplemented to the culture medium. Subsequently, the mixture was incubated for $90 \mathrm{~min}$ at $37^{\circ} \mathrm{C}$, and the absorbance was detected at $450 \mathrm{~nm}$ using a spectrophotometer (BioTek Instruments, Inc., Winooski, VT, USA). Relative cell viability (\%) = (absorbance at $450 \mathrm{~nm}$ of the treated group-absorbance at $450 \mathrm{~nm}$ of the blank) / (absorbance at 450 $\mathrm{nm}$ of the control group-absorbance at $450 \mathrm{~nm}$ of the blank) $\times 100 \%$.

\section{Colony formation assay.}

Transfected cells were planted into 6-well plates and maintained for 14 days at $37^{\circ} \mathrm{C}$. Subsequently, cells were washed with PBS and fixed with $4 \%$ paraformaldehyde. The cells were then stained with $0.1 \%$ crystal violet (Sigma-Aldrich; Merck KGaA, Darmstadt, Germany). The stained colonies were viewed and calculated using a fluorescence microscope.

\section{Apoptosis analysis.}

For apoptosis analysis, transfected cells were seeded and maintained for $48 \mathrm{~h}$ and then collected and washed twice with PBS and resuspended in buffer. Cell apoptosis assay was conducted by using annexin 
V-fluorescein isothiocyanate apoptosis detection kit (cat. no. 556547; BD Biosciences) in accordance to the manufacturer's protocol.

\section{Luciferase reporter assay.}

Cells were cultured to $60-70 \%$ confluence for transfection. Circ-PAN3-WT vector or Circ-PAN3-mutant (MUT) vector were created with or without a 3'-untranslated region binding site for miR-153 using pmirGLO vector (Promega Corporation) and CCDN1-WT vector or CCDN1-MUT vector were constructed similarly. Cells were co-transfected with miRNA mimics or NC mimics along with luciferase reporter vector using Lipofectamine 3000 (cat. no. L3000008; Invitrogen; Thermo Fisher Scientific, Inc.). Following transfection for $48 \mathrm{~h}$, luciferase activity was detected with a Dual-Luciferase Reporter Assay System (cat. no. E1910; Promega Corporation).

\section{RNA pull-down assay}

HCC cells were transfected, harvested, lysed and sonicated. The circ-PAN3 probe was used for incubation with C-1 magnetic beads (Life Technologies) at $25^{\circ} \mathrm{C}$ for $2 \mathrm{~h}$ to generate probe-coated beads. Cell lysate with circ-PAN3 probe or oligo probe was incubated at $4{ }^{\circ} \mathrm{C}$ for one night. After washing with wash buffer, the RNA mix bound to the beads was eluted and extracted with a RNeasy Mini Kit (QIAGEN) for subsequent RT-qPCR.

\section{Protein extraction and western blot analysis.}

Proteins were isolated using RIPA buffer (cat. no. P0013B; Beyotime Institute of Biotechnology) supplemented with EDTA-free protease inhibitor cocktail (cat. no. 04693159001; Roche Diagnostics $\mathrm{GmbH}$ ) as described previously. The extracted proteins were separated using $10 \%$ sodium dodecyl sulfate-polyacrylamide gel electrophoresis, then transferred to PVDF membranes. Then the PVDF membranes were blocked with $5 \%$ skimmed milk. Subsequently, the membranes were incubated with primary antibodies at a dilution ratio of 1:1000 with PBST containing $5 \%$ BSA overnight at $4^{\circ} \mathrm{C}$. Next, the membranes were incubated with horseradish peroxidase-labeled secondary antibodies at dilution ratio of 1:5000 for $1 \mathrm{~h}$ at room temperature. Enhanced chemiluminescence (cat. no. 407207; EMD Millipore; Merck KGaA) was used to visualize the target proteins using the Tanon 4600 imaging system (Tanon Science and Technology Co., Ltd.). The antibodies used include Cyclin D1 (cat. no. 55506S; Cell Signaling Technology, Inc.), GAPDH (no. ab128915; Abcam).

\section{HCC cancer xenograft model.}

All the experiments concerning animals were reviewed and approved by the Animal Welfare Committee of Affiliated Tongji Hospital, Tongji Medical College, Huazhong University of Science and Technology. BALB/c nude mice (4 weeks old) were purchased from Model Animal Research Center of Nanjing University (Nanjing, China). Transfected Huh7 cells $\left(3 \times 10^{\wedge} 6\right)$ in serum-free DMEM medium were injected into the right flank of the nude mice. After 4 weeks, tumor volume $(V)$ was calculated using the following formula: $V=$ length $\mathrm{x}$ width $2 / 2$ and tumor weight was measured. 


\section{Statistical analysis.}

All data generated by above procedures were statistically analyzed using the GraphPad Prism v8.0 (GraphPad Software, Inc.). Survival curves were produced using the Kaplan-Meier method, and a log-rank test was used for comparison. The correlation between the expression levels of two genes was analyzed using Pearson's correlation analysis. Student's t test, $\chi^{2}$ test or one-way ANOVA was adopted appropriately. $\mathrm{P}<0.05$ was considered to exhibit a significant difference statistically. Data are presented as mean $\pm \mathrm{SEM}$ from three independent experiments.

\section{Results}

\section{Circ-PAN3 is frequently overexpressed in HCC and associates with poor prognosis}

To investigate the expression pattern of circ-PAN3 in HCC, the levels of circ-PAN3 in $80 \mathrm{HCC}$ tumor tissues and 80 adjacent normal tissues was detected. Consistant with the studies in other malignancy, the expression level of circ-PAN3 was significantly elevated in HCC tumor tissues compared with that in adjacent normal tissues (Fig. 1A; $p<0.001$ ). Then, the expression level of circ-PAN3 was measured in HCC cell lines (Huh7, HCCLM3, SK-HEP-1) and normal liver cell LO2. The results showed that the level of circ-PAN3 in HCC cell lines was statistically higher than that in normal liver cell LO2 (Fig. 1B; $p<0.001$ ). Moreover, the association of circ-PAN3 level and clinical characteristics was analyzed. The expression of circ-PAN3 is statistically associated with tumor size, TNM staging, and lymph node metastasis, but the association between circ-PAN3 level and age, sex or tumor differentiation was marginal (Table 1). Next, the association between circ-PAN3 expression level and clinical outcomes of HCC patients was explored. The results showed that elevated expression of circ-PAN3 was significantly associated with poor overall survival time (Fig. 1C; $p<0.001$ ).

\section{Knockdown of circ-PAN3 inhibitsHCC cell proliferation in vitro as well as tumor growth in vivo}

We further elucidate the functional mechanisms of circ-PAN3 in HCC in vitro and in vivo. Two cell lines of high-level circ-PAN3 were selected to be transfected with si-circ-PAN3 to knockdown the expression level of circ-PAN3 (Fig. 2A). Following transfection, the cell viability, colony formation and cell proliferation assays were conducted. The results exhibited that cell proliferation and colony formation were validated to be significantly inhibited after transfection with si-circ-PAN3 in SK-Hep-1 and Huh7 cells (Fig. 2B-C). Further, cell apoptosis analysis was performed and indicated that downregulation of circ-PAN3 caused an apparent increase in apoptosis rate of HCC cells, when compared with that in the control groups (Fig. 2D). Together, these results manifested that downregulation of circ-PAN3 suppressed HCC cell growth through induction of cell apoptosis in vitro.

Next, we clarified the function of circ-PAN3 using HCC tumor xenograft models. The results showed that injection with si-circ-PAN3 inhibited tumor growth compared to the si-nc groups significantly (Fig. 2E). Altogether, these data revealed that the downregulation of circ-PAN3 could significantly induce inhibit HCC proliferation in vivo. 


\section{Circ-PAN3 was positively correlated with cyclin D1 expression in HCC cells}

Since cyclin D1 was upregulated in several malignancies with high expression level of circ-PAN3, we explored the relationship of circ-PAN3 and cyclin D1. Our results showed that circ-PAN3 knockdown downregulated the expression of cyclin D1, but induced merely marginal alteration of other cell cycleassociated genes in SK-Hep-1 and Huh7 cells (Fig. 3A). Moreover, the expression levels of cyclin D1 was found to be upregulation in HCC cell lines with high expression of circ-PAN3 (Fig. 3B \& 3C). The expression level of cyclin D1 was significantly higher in HCC tumor tissues compared with that in adjacent normal tissues (Fig. 3D). Pearson's correlation analysis indicated the positive correlation between the expression levels of circ-PAN3 and cyclin D1 (Fig. 3E). These results showed that circ-PAN3 expression was positively correlated with cyclin D1 in HCC, which indicated the possible regulatory role of circ-PAN3 on cyclin D1.

\section{Circ-PAN3 sponged miR-153 to regulate cyclin D1 in HCC cells}

To further investigate the regulatory mechanisms of circ-PAN3 on cyclin D1, the following experiments were performed. Since circ-RNA function could be largely depended on subcellular localization, we analyzed subcellular RNA level to identify the predominant location of circ-PAN3. This analysis showed that circ-PAN3 was mostly located in cytoplasm in SK-Hep-1 and Huh7 cells (Fig. 4A). Previous studies have been reported that circRNAs could act as miRNA sponges. Thus, Circlnteractome (https://circinteractome.nia.nih.gov/) and Targetscan (http://targetscan.org/vert_72) were used to identify the possible interaction between circ-PAN3 and cyclin D1. According to the prediction, circ-PAN3 could bind and sponge miR-153, which then in turn targeted cyclin D1 to accelerate the growth of HCC cells (Fig. 4B). To verify the prediction, luciferase reporter assays were conducted to confirm the predictive interaction among circ-PAN3, miR-153, and cyclin D1. In accordance to the prediction, the results indicated that miR-153 significantly reduced the luciferase activity in circ-PAN3-WT vector but not in circPAN3-MUT vector in SK-Hep-1 and Huh7 cells (Fig. 4C). In addition, miR-153 Iso reduced the luciferase activity in CCND1-WT vector but not in CCND1-MUT vector significantly in SK-Hep-1 and Huh7 cells (Fig. 4D). Moreover, RNA pull-down with biotinylated-circ-PAN3 probe confirmed direct interaction between circPAN3 and miR-153 in SK-Hep-1 and Huh7 cells (Fig. 4E). RNA pull-down with biotinylated-CNND1 probe also validated direct interaction between CNND1 and miR-153 in SK-Hep-1 and Huh7 cells (Fig. 4E). Next, we detect the expression level of miR-153 following circ-PAN3 knock-down. We found knock-down of circPAN3 significantly elevated the expression of miR-153 (Fig. 4F). We then evaluated the expression level of cyclin D1 with downregulation of circ-PAN3. The results unveiled that downregulation of circ-PAN3 reduced the expression of cyclin D1 in SK-Hep-1 and Huh7 cells (Fig. 4F). Since cyclin D1 was a direct target of miR-153, we evaluated the expression level of cyclin D1 with upregulation of miR-153. The results showed that overexpression of miR-153 also reduced the expression of cyclin D1 in RNA and protein level (Fig. 4G). Together, these results indicated that circ-PAN3 positively regulated expression of cyclin D1 via sponging miR-153. 


\section{Silencing of miR-153 or overexpression of cyclin D1 rescues the decreased proliferative abilities of HCC cells caused by circ-PAN3 knockdown}

To further clarify the relationship between circ-PAN3, miR-153 and cyclin D1, rescue analysis was conducted. The results showed that the inhibitory expression of cyclin D1 following circ-PAN3 knockdown was reversed with the addition of miR-153 inhibitor or cyclin D1 plasmid (Fig. 5A \& 5B). We then evaluated the cell phenotypes with rescue experiments. The results showed circ-PAN3 knockdown impaired cell proliferation and cell colony formation (Fig. 5C \& 5D). Notably, the inhibition of cell proliferation and colony formation were recovered following the combination with miR-153 inhibitor, (Fig. $5 C \& 5 D)$. Moreover, the suppression of cell proliferation and colony formation could be reversed following the combination with cyclin D1 plasmid (Fig. 5C \& 5D). Combined with the results above, these results demonstrated the circ-PAN3/miR-153/cyclin D1 modulated the growth of HCC cells.

\section{Discussion}

CircRNAs are wildly reported to be involved in the tumorigenesis and progression of various types of cancers, but the role of circRNAs in HCC remains to be investigated further. In this study, it was revealed that circ-PAN3, was statistically increased in HCC clinical tissues and associated with poor survival rate. The experimental data revealed that circ-PAN3 served as a competitive endogenous RNA to deteriorate miR-153 activity. Impairment of miR-153 activity would then upregulate cyclin D1 and promote the proliferation of HCC cell. Interestingly, it was also demonstrated that overexpression of cyclin D1 reversed the inhibition of HCC proliferation induced by reducing circ-PAN3 expression. Taken together, it has been established that circ-PAN3 might be used as a promising diagnostic and therapeutic target in HCC.

Circ-PAN3 is a newly identified circRNA, which is located on 13q12.2 [17]. In recent years, several studies have investigated the role of circ-PAN3 in various diseases [12, 13]. In acute myeloid leukemia, circ-PAN3 promoted drug resistance through regulation of autophagy as an autophagy inducer via the AMPK/mTOR pathway [12]. In another study of acute myeloid leukemia, circ-PAN3 was demonstrated to be a key mediator for chemo-resistance of AML cells through circ-PAN3-miR-153-5p/miR-183-5p-XIAP regulatory axis [17]. In cardiac fibrosis, circ-PAN3 exhibited profibrotic effects via miR-221/FoxO3/ATG7 axis [18]. However, the role and potential mechanisms of circ-PAN3 in HCC remains largely unknown. In this study, two cell lines (SK-Hep-1 and Huh7) were selected with significantly high expression of circPAN3 from four common HCC cell lines to investigate the possible function of circ-PAN3. It was showed that knockdown of circ-PAN3 significantly impaired HCC cell proliferation and colony formation. It was further revealed that circ-PAN3 knockdown induced HCC cell apoptosis significantly. These results suggest that circ-PAN3 functioned as a promoter in HCC cell proliferation.

CircRNAs can act through diverse mechanisms, including genomic targeting, regulation in cis or trans, and antisense interference [5]. In recent years, the role of circRNA as competitive endogenous RNA in multiple types of cancer has gained much attention and has been broadly reported $[8,10]$. The competitive endogenous RNA can decrease the stability of target miRNA, thereby upregulating the 
expression of the miRNA target gene. In the present study, the potential miRNA that circ-PAN3 might regulate in HCC was investigated. It was showed that circ-PAN3 contained the binding site of miR-153 using bioinformatic analysis. The interaction between circ-PAN3 and miR-153 was further demonstrated using luciferase assay and RIP. Moreover, circ-PAN3 knockdown increased the expression of miR-153. In addition, cell apoptosis induced by circ-PAN knockdown could be reversed by miR-153 inhibitor. These results indicate that circ-PAN3 might exert oncogenic function by inhibiting the expression of miR-153.

Multiple studies have reported that cyclin D1 plays a pro-tumorigenic role in HCC and downregulation of cyclin D1 was correlated with reduced cell proliferation and invasion $[19,20]$. The expression of cyclin D1 could be regulated by various mechanisms including autophagy degradation system and miRNA inhibition [21]. Previous studies reported that cyclin D1 could be positively regulated by X-linked inhibitor of apoptosis protein (XIAP) through E3 ligase-mediated phosphorylation [16]. Notably, circ-PAN3 could regulate positively XIAP to induce chemo-resistance in acute myeloid leukemia. This indicated a potential positive association between circ-PAN3 and cyclin D1 [17]. In the present study, we investigated the regulatory role of circ-PAN3 in cyclin D1. We found that circ-PAN3 could modulate the expression of cyclin D1 via miRNA sponge. Targetscan database was used to explore potential targets that could bind with miR-153. It was found that cyclin D1 directly interacted with miR-153 and this was validated using a luciferase assay and cyclin D1 expression was downregulated by miR-153. Subsequently, it was found that downregulation of circ-PAN3 could decrease cyclin D1 expression and this effect could be reversed by the addition of miR-153 inhibitor. Finally, it was confirmed that the inhibition of HCC cell proliferation induced by circ-PAN3 knockdown could be rescued by the overexpression of cyclin D1. In total, it was demonstrated that the novel circRNA, circ-PAN3, could promote the progression of HCC through the circPAN3/miR-153/cyclin D1 axis.

\section{Conclusions}

In conclusion, it was revealed that circ-PAN3 was increased in HCC tumor tissues and might be used as a predictive marker of prognosis. Notably, to the best of our knowledge this is the first study to establish that the role of circ-PAN3 could accelerate the progression of HCC through the miR-153/cyclin D1 pathway. Thus, circ-PAN3 could be adopted as a novel diagnostic and therapeutic target in the management of HCC.

\section{Declarations}

\section{Ethics approval and consent to participate}

The protocol of this study was reviewed and approved by the Ethics Committee of Medical Research, Affiliated Tongji Hospital, Tongji Medical College, Huazhong University of Science and Technology. Patients provided written informed consent for the use of tumor and adjacent tissues at Affiliated Tongji Hospital, Tongji Medical College, Huazhong University of Science and Technology. 


\section{Consent for publication}

Written informed consent for publication was obtained from all participants.

\section{Availability of data and materials}

Not applicable.

\section{Competing interests}

The authors declare that they have no competing interests.

\section{Funding}

This study was funded by The National Natural Science Foundation of China (Grants No. 81272659 to Renyin Qin, 81602475 to Xingjun Guo, 81502633 to Xu Li).

\section{Authors' contributions}

$\mathrm{RQ}$ designed the study. SY, MW, XL performed the cell experiments. SY, MW and XG conducted the in vivo experiments. SY and RQ drafted the manuscript. All authors read and approved the final manuscript for publication.

\section{Acknowledgements}

Not applicable.

\section{Reference}

1. Bray, F., et al., Global cancer statistics 2018: GLOBOCAN estimates of incidence and mortality worldwide for 36 cancers in 185 countries. CA Cancer J Clin, 2018. 68(6): p. 394-424.

2. Siegel, R.L., K.D. Miller, and A. Jemal, Cancer statistics, 2020. CA Cancer J Clin, 2020. 70(1): p. 7-30.

3. Roayaie, S., et al., The role of hepatic resection in the treatment of hepatocellular cancer. Hepatology, 2015. 62(2): p. 440-51.

4. Villanueva, A., Hepatocellular Carcinoma. N Engl J Med, 2019. 380(15): p. 1450-1462.

5. Meng, S., et al., CircRNA: functions and properties of a novel potential biomarker for cancer. Mol Cancer, 2017. 16(1): p. 94.

6. Kristensen, L.S., et al., The biogenesis, biology and characterization of circular RNAs. Nat Rev Genet, 2019. 20(11): p. 675-691. 
7. Zhang, $\mathrm{H}$., et al., Exosome circRNA secreted from adipocytes promotes the growth of hepatocellular carcinoma by targeting deubiquitination-related USP7. Oncogene, 2019. 38(15): p. 2844-2859.

8. Wang, Y.G., et al., hsa_circ_0091570 acts as a ceRNA to suppress hepatocellular cancer progression by sponging hsa-miR-1307. Cancer Lett, 2019. 460: p. 128-138.

9. Liu, Y., et al., Long non-coding RNA HULC activates HBV by modulating HBX/STAT3/miR539/APOBEC3B signaling in HBV-related hepatocellular carcinoma. Cancer Lett, 2019. 454: p. 158-170.

10. Han, D., et al., Circular RNA circMTO1 acts as the sponge of microRNA-9 to suppress hepatocellular carcinoma progression. Hepatology, 2017. 66(4): p. 1151-1164.

11. Wang, H., et al., CircSLC3A2 functions as an oncogenic factor in hepatocellular carcinoma by sponging miR-490-3p and regulating PPM1F expression. Mol Cancer, 2018. 17(1): p. 165.

12. Shang, J., et al., CircPAN3 contributes to drug resistance in acute myeloid leukemia through regulation of autophagy. Leuk Res, 2019. 85: p. 106198.

13. Zhu, P., et al., IL-13 secreted by ILC2s promotes the self-renewal of intestinal stem cells through circular RNA circPan3. Nat Immunol, 2019. 20(2): p. 183-194.

14. Musgrove, E.A., et al., Cyclin D as a therapeutic target in cancer. Nat Rev Cancer, 2011. 11(8): p. 55872 .

15. Chalermrujinanant, C., et al., Cyclin D1 promotes BRCA2-Rad51 interaction by restricting cyclin A/Bdependent BRCA2 phosphorylation. Oncogene, 2016. 35(22): p. 2815-23.

16. Cao, Z., et al., X-linked inhibitor of apoptosis protein (XIAP) regulation of cyclin D1 protein expression and cancer cell anchorage-independent growth via its E3 ligase-mediated protein phosphatase $2 A / c-J u n$ axis. J Biol Chem, 2013. 288(28): p. 20238-47.

17. Shang, J., et al., CircPAN3 mediates drug resistance in acute myeloid leukemia through the miR-1535p/miR-183-5p-XIAP axis. Exp Hematol, 2019. 70: p. $42-54$ e3.

18. Li, F., et al., circPAN3 exerts a profibrotic role via sponging miR-221 through FoxO3/ATG7-activated autophagy in a rat model of myocardial infarction. Life Sci, 2020. 257: p. 118015.

19. Kitisin, K., et al., Disruption of transforming growth factor-beta signaling through beta-spectrin ELF leads to hepatocellular cancer through cyclin D1 activation. Oncogene, 2007. 26(50): p. 7103-10.

20. Jung, Y.J., et al., Reciprocal expressions of cyclin E and cyclin D1 in hepatocellular carcinoma. Cancer Lett, 2001. 168(1): p. 57-63.

21. Wu, S.Y., et al., Hepatocellular carcinoma-related cyclin D1 is selectively regulated by autophagy degradation system. Hepatology, 2018. 68(1): p. 141-154 


\section{Tables}

Table 1 Association between expression of circ-PAN3 and clinical stage of HCC

\begin{tabular}{|c|c|c|c|}
\hline \multirow[t]{2}{*}{ Variable } & \multicolumn{2}{|c|}{ circ-PAN3 } & \multirow[t]{2}{*}{$P$ value } \\
\hline & Low & High & \\
\hline \multicolumn{4}{|l|}{ Age(years) } \\
\hline$\leq 50$ & 19 & 23 & 0.502 \\
\hline$\bigotimes 50$ & 21 & 17 & \\
\hline \multicolumn{4}{|l|}{ Sex } \\
\hline Female & 16 & 11 & 0.344 \\
\hline Male & 24 & 29 & \\
\hline \multicolumn{4}{|l|}{ Tumor size } \\
\hline$\leq 5$ & 24 & 11 & 0.007 \\
\hline$\nabla 5$ & 16 & 29 & \\
\hline \multicolumn{4}{|l|}{ TNM } \\
\hline I/ II & 21 & 10 & 0.021 \\
\hline III/IV & 19 & 30 & \\
\hline \multicolumn{4}{|c|}{ Lymph node metastasis } \\
\hline Yes & 11 & 26 & 0.002 \\
\hline No & 29 & 14 & \\
\hline \multicolumn{4}{|c|}{ Tumor differentiation } \\
\hline High & 8 & 16 & 0.086 \\
\hline Middle & 13 & 11 & \\
\hline Low & 19 & 11 & \\
\hline
\end{tabular}

Figures 
A

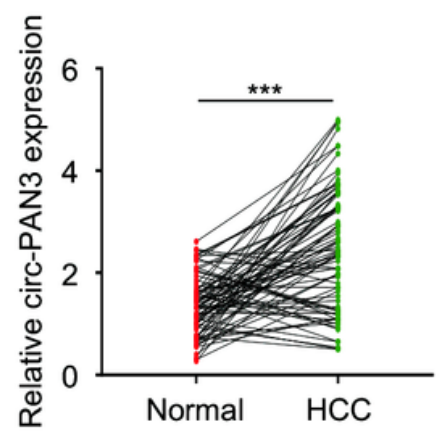

B

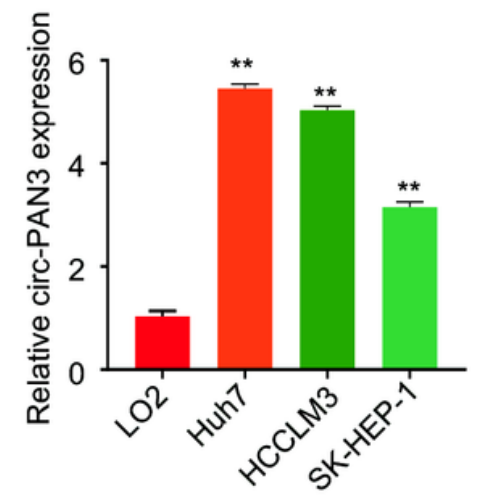

C

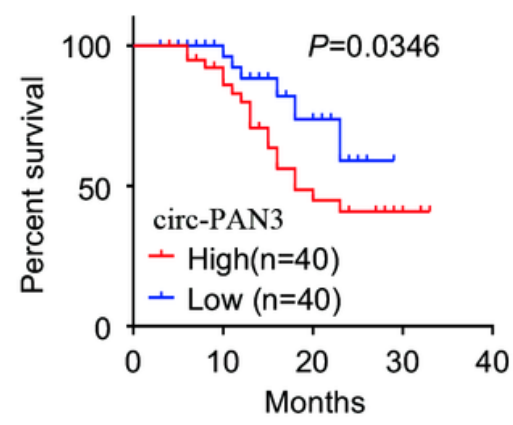

\section{Figure 1}

circ-PAN3 expression in HCC tissues and cell lines and its correlation with clinical outcomes. (A) Expression level of circ-PAN3 in HCC tissues $(n=80)$ compared with adjacent normal tissues $(n=80)$ was measured using qPCR. (B) circ-PAN3 expression was analyzed in HCC cell lines (Huh7, HCCLM3, SK-HEP1) and human normal liver cell (LO2). (C) Kaplan-Meier curves for overall survival in patients with HCC with high $(n=40)$ and low circ-PAN3 expression level $(n=40)$. Data are presented as mean $\pm S E M ; n \geq 3$. ${ }^{*} \mathrm{P}<0.05 ;{ }^{*} \mathrm{P}<0.01$. HCC, Hepatocellular cancer. 
A

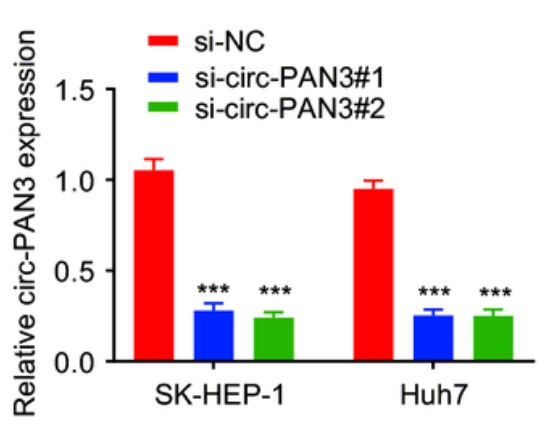

B

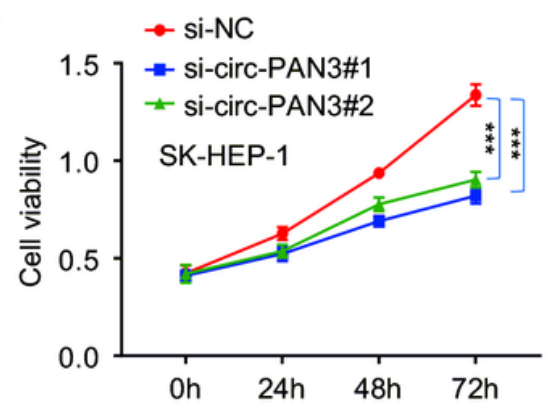

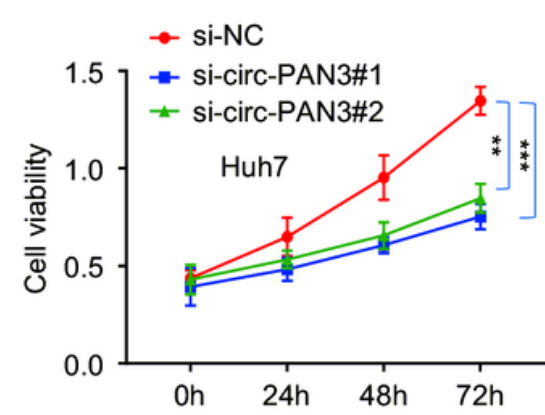
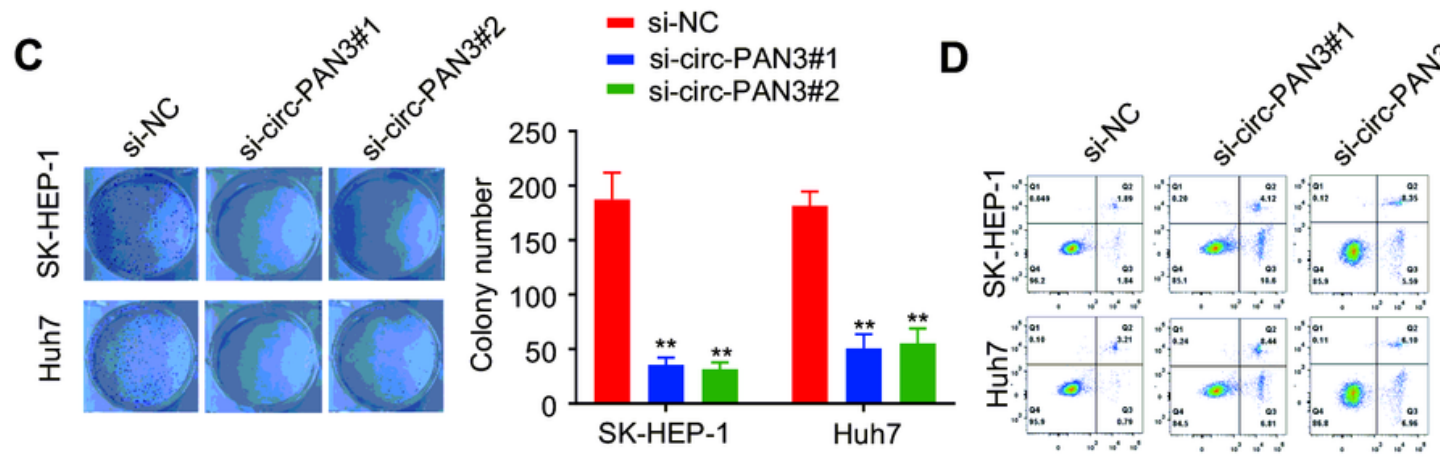

- si-NC

- si-circ-PAN3\#1

- si-circ-PAN3\#2

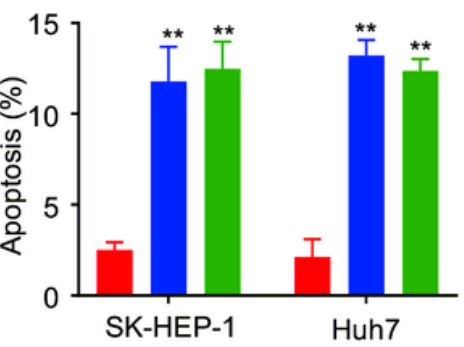

E

si-NC
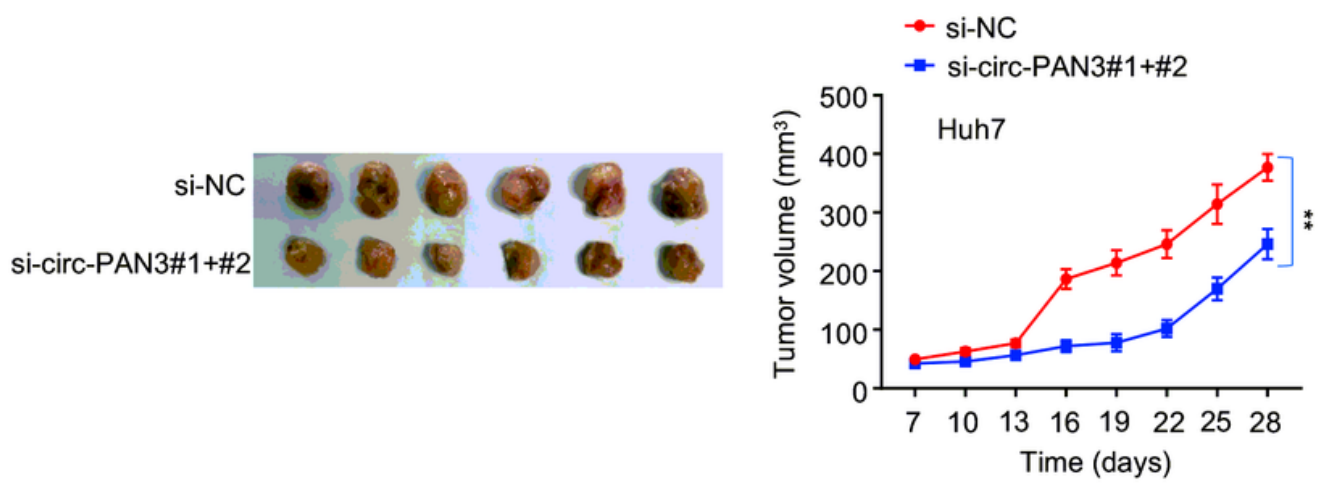

- si-NC

- si-circ-PAN3\#1+\#2

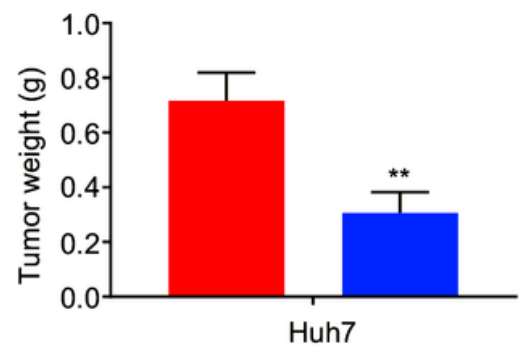

\section{Figure 2}

Effect of circ-PAN3 on cell proliferation and colony formation of HCC in vitro and in vivo. (A) circ-PAN3 was inhibited by circ-PAN3 siRNAs in SK-Hep-1 and Huh7 cells, and expression level of circ-PAN3 was measured using qPCR. (B) Cell viability was quantified using Cell Counting Kit-8 assays after transfecting circ-PAN3 siRNAs in SK-Hep-1 (left) and Huh7 (right) cells. (C) Colony formation assays were performed after transfecting circ-PAN3 siRNAs in SK-Hep-1 and Huh7 cells for 14 days (left) and cell colony numbers were quantified (right). (D) Cell apoptosis was detected using flow cytometry (left) and the percentage of apoptotic cells was quantified (right) in SK-Hep-1 and Huh7 cells transfected with circ-PAN3 siRNAs. (E) Huh7 cells transfected with circ-PAN3 siRNAs or negative control were injected subcutaneously into the flanks of nude mice. Tumors were extracted after 4 weeks and photographed (left). Tumor volumes of 
mice were measured after 1 week (middle). The tumor weights of mice were compared among the groups after 4 weeks (right). Data are presented as mean $\pm S E M ; n \geq 3 .{ }^{*} P<0.05 ; * \star P<0.01 ; * \star * P<0.001$.
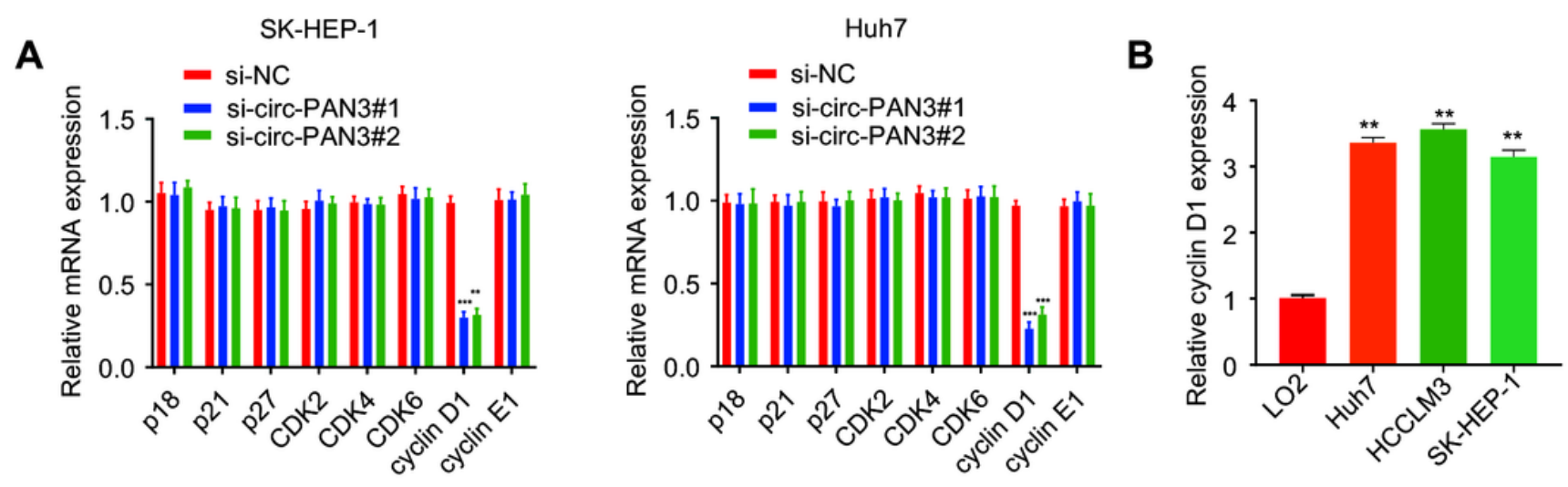

C

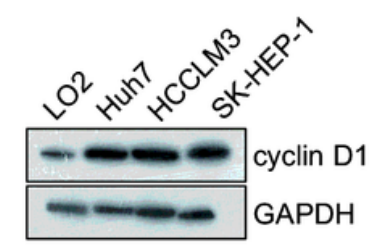

D

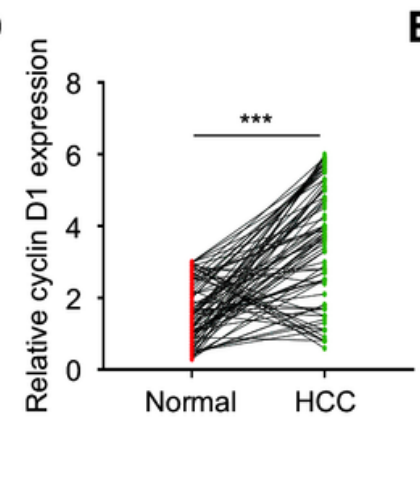

E

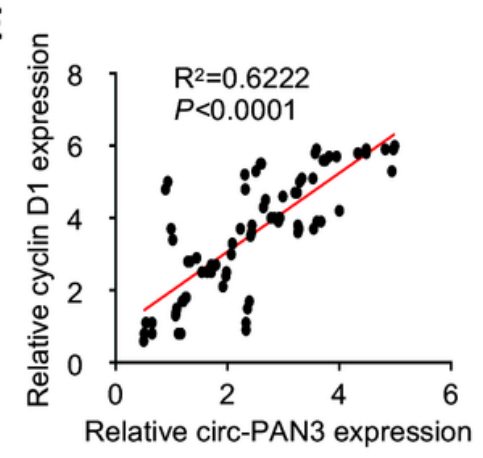

Figure 3

Circ-PAN3 elevated cyclin D1 expression in HCC cells. (A) The expression levels of cell cycle associated genes in SK-Hep-1 (left) and Huh7 (right) cells transfected with circ-PAN3 siRNAs. (B) The expression levels of cyclin D1 in HCC cell lines (Huh7, HCCLM3, SK-HEP-1) and human normal liver cell (LO2) were measured using qPCR. (C) The expression levels of cyclin D1 in HCC cell lines (Huh7, HCCLM3, SK-HEP-1) and human normal liver cell (LO2) were evaluate using western blot analysis (left) and were quantified (right). (D) Expression level of cyclin D1 in HCC tissues $(n=80)$ compared with adjacent normal tissues $(n=80)$ was measured using qPCR. (E) The correlation between circ-PAN3 and cyclin D1 expression levels was analyzed using Pearson's correlation analysis. Data are presented as mean $\pm S E M ; n \geq 3$. ${ }^{*}<0.05$; **P<0.01; ***P<0.001. 
A

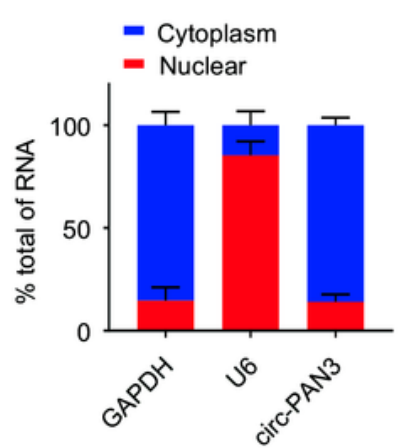

C

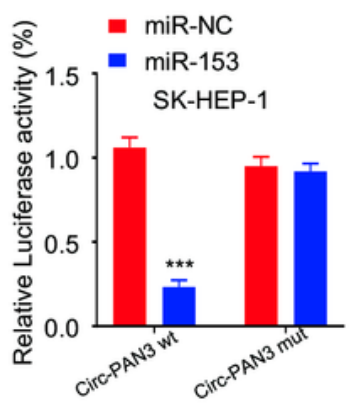

E
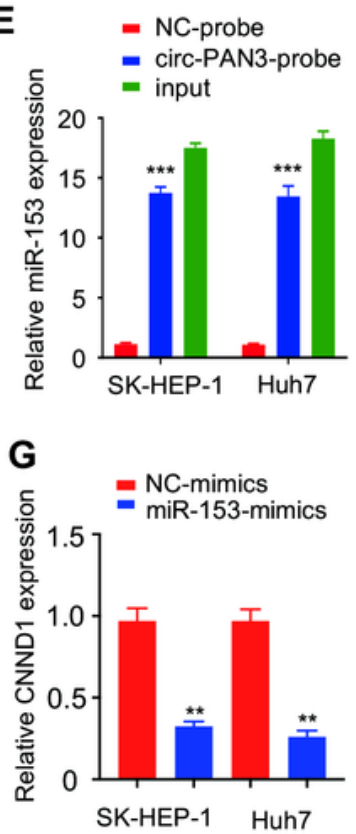

B

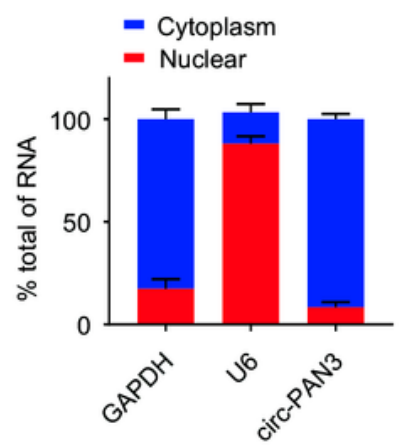

D
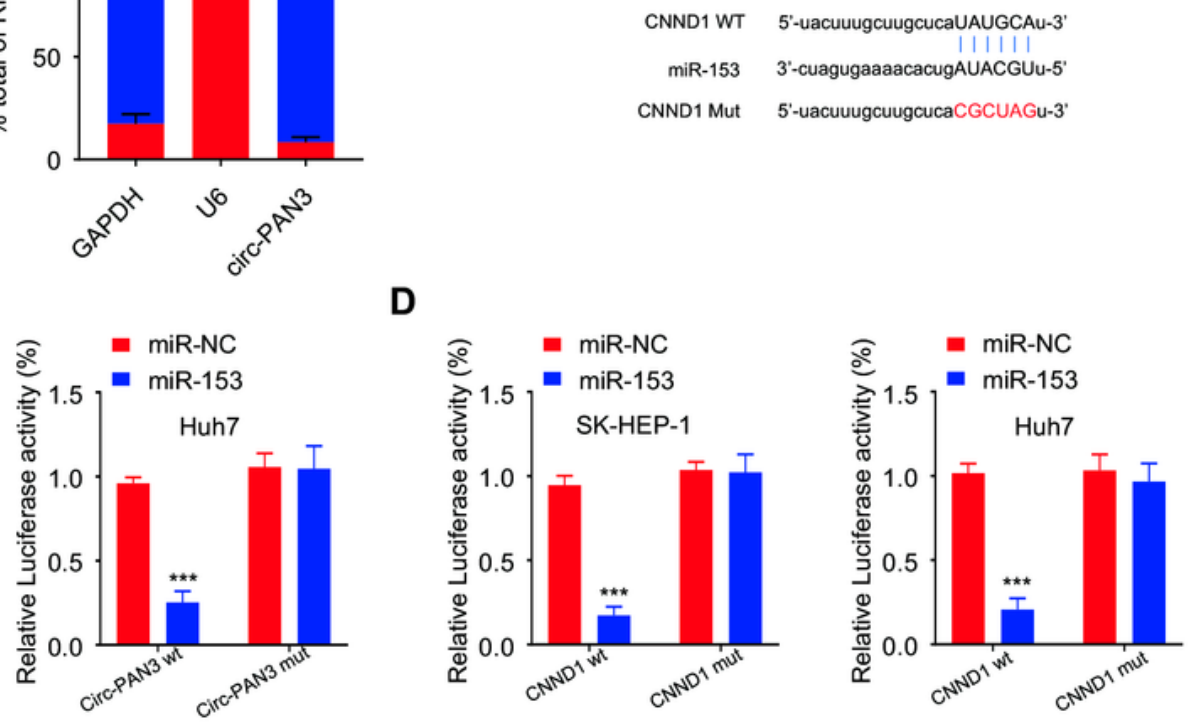

CirC-PAN3 WT 5'-ACCUCACGUUGCUUAUAUGCAAC-3'

miR-153 3'-CUAGUGAAAACACUGAUACGUU-5

CirC-PAN3 Mut 5'-ACCUCACGUUGCUUAGCGUAGGC-3'

CNND1 Mut 5'-uacuuugcuugcucaCGCUAGu-3'

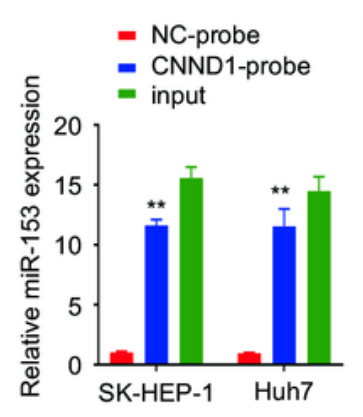

$\mathbf{F}$
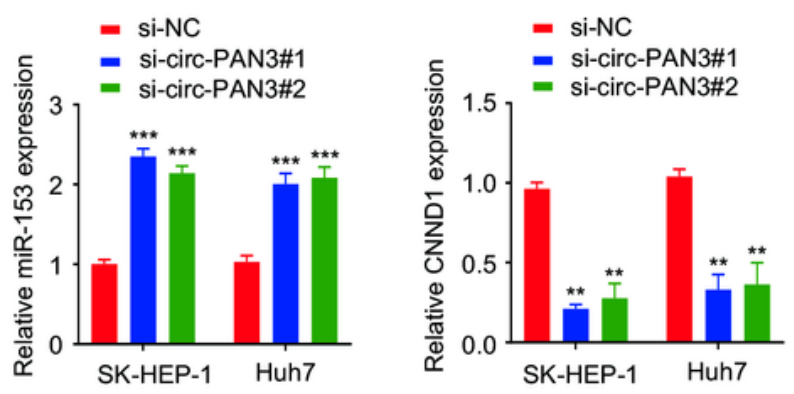

\section{Figure 4}

Circ-PAN3 sponged to regulate cyclin D1 in HCC cells. (A) qRT-PCR analysis of circ-PAN3 RNA in either the cytoplasm or the nucleus in SK-HEP-1 (left) and Huh7 (right) cells. (B) Potential binding region between circ-PAN3 and miR-153 (upper) was predicted using Circlnteractome database, and potential binding region between cyclin D1 and miR-153 (lower) was predicted using Targetscan database. (C) MiR-153 was validated as direct targets of circ-PAN3 using dual luciferase reporter assays in SK-HEP-1 (left) and 
Huh7 (right) cells. (D) Cyclin D1 was validated as a direct target of miR-153 using dual luciferase reporter assays in SK-HEP-1 (left) and Huh7 (right) cells. (E) RNA pull-down with biotinylated-circ-PAN3 probe confirmed direct interaction between circ-PAN3 and miR-153 in SK-HEP-1 and Huh7 cells (left). RNA pulldown with biotinylated-circ-CNND1 probe confirmed direct interaction between circ-PAN3 and miR-153 in SK-HEP-1 and Huh7 cells (right). (F) The expression levels of miR-153 (left) and cyclin D1 (right) in SKHEP-1 and Huh7 cells transfected with circ-PAN3 siRNAs. (G) The expression levels of cyclin D1 in cells transfected with miR-153 mimics in SK-HEP-1 and Huh7 cells. Data are presented as mean \pm SEM; $n \geq 3$. ${ }^{*} P<0.05 ; * \star P<0.01 ; * \star * P<0.001$. 


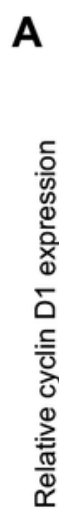

- si-NC

- si-Cir-PAN3\#1

- si-circ-PAN3\#1+inh-miR-153

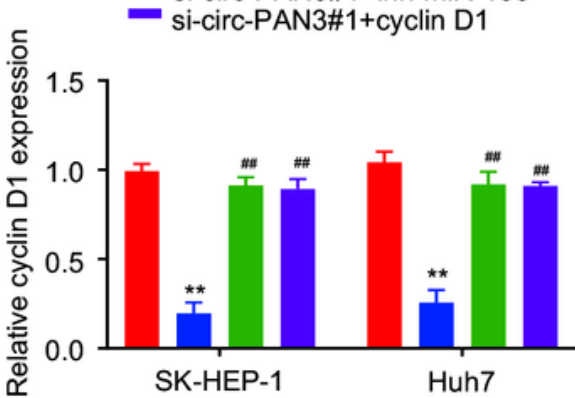

C

$$
\begin{aligned}
& \rightarrow \text { si-NC } \\
& \neq \text { si-circ-PAN3\#1 } \\
& \neq \text { si-circ-PAN3\#1+inh-miR-153 } \\
& \rightarrow \text { si-circ-PAN3\#1+cyclin D1 }
\end{aligned}
$$

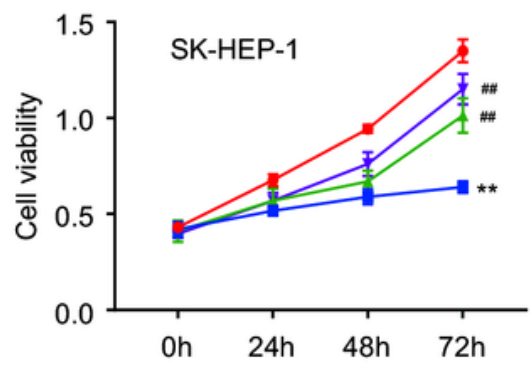

D

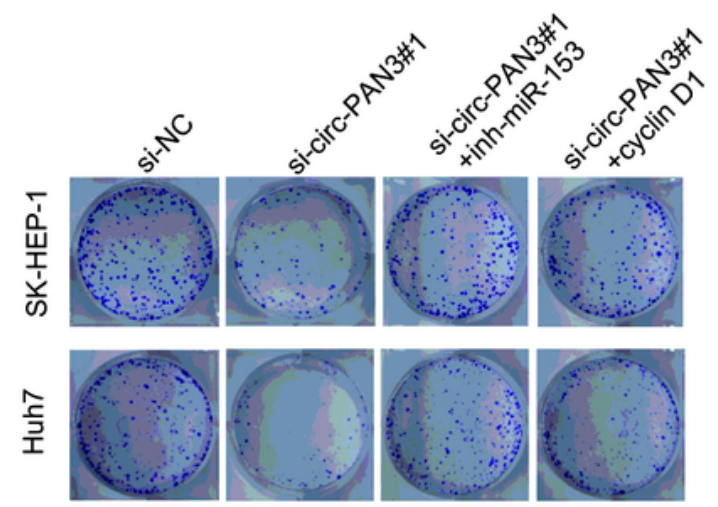

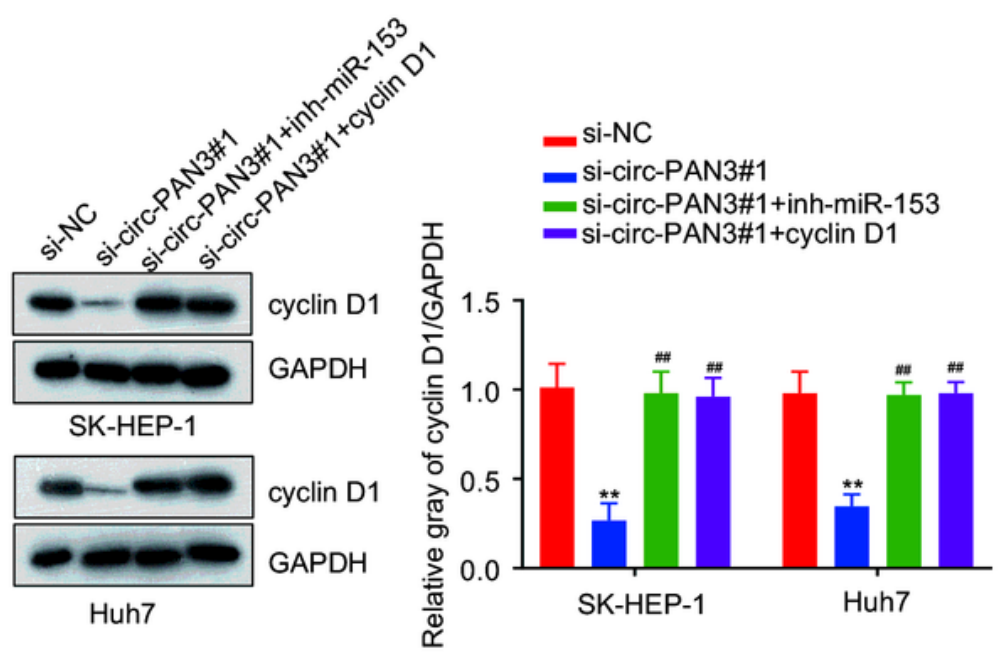

$\rightarrow$ si-NC

- si-circ-PAN3\#1

\# si-circ-PAN3\#1+inh-miR-153

* si-circ-PAN3\#1+cyclin D1

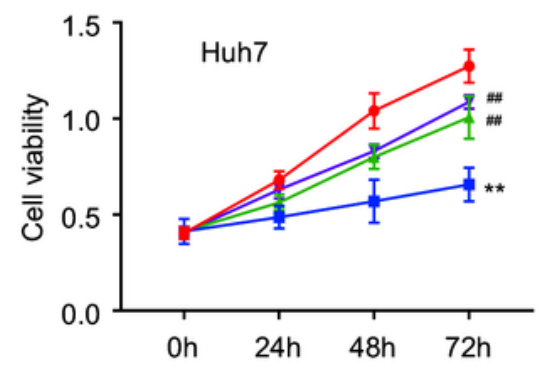

- si-NC

- si-circ-PAN3\#1

- si-circ-PAN3\#1+inh-miR-153

- si-circ-PAN3\#1+cyclin D1

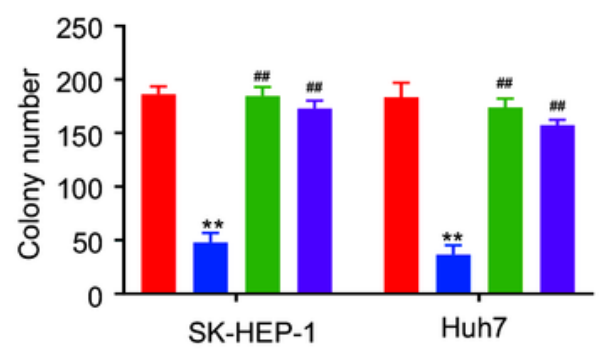

\section{Figure 5}

Circ-PAN3/miR153/cyclin D1 axis in HCC cells. (A) The expression of cyclin D1 was measure by qRT-PCR after transfecting circ-PAN3-siRNA, circ-PAN3-siRNA and miR-153 inhibitor, circ-PAN3-siRNA and cyclin D1 plasmid in SK-HEP-1 and Huh7 cells. (B) The expression of cyclin D1 was measured (left) and quantified (right) by western blotting after transfecting circ-PAN3-siRNA, circ-PAN3-siRNA and miR-153 inhibitor, circPAN3-siRNA and cyclin D1 plasmid in SK-HEP-1 and Huh7 cells. (C) Cell viability was quantified using 
Cell Counting Kit-8 assays after transfecting circ-PAN3-siRNA, circ-PAN3-siRNA and miR-153 inhibitor, circPAN3-siRNA and cyclin D1 plasmid in SK-HEP-1 (left) and Huh7 (right) cells. (D) Colony formation assays were performed after transfecting circ-PAN3-siRNA, circ-PAN3-siRNA and miR-153 inhibitor, circ-PAN3siRNA and cyclin D1 plasmid in SK-HEP-1 (left) and Huh7 (right) cells. Data are presented as mean \pm SEM; $n \geq 3$. *P<0.05; ** $\mathrm{P}<0.01 ; * \star * P<0.001$. 